\title{
MicroRNA-433 targets AKT3 and inhibits cell proliferation and viability in breast cancer
}

\author{
XIAOLEI HU ${ }^{1 *}$, JIE WANG $^{2 *}$, WAN HE $^{3 *}$, PAN ZHAO $^{4}$ and CHANGSHENG YE ${ }^{1}$ \\ ${ }^{1}$ Department of Breast Surgery, Nanfang Hospital, Southern Medical University, Guangzhou, Guangdong 510515; \\ ${ }^{2}$ Clinical Nutrition Department, The 458th Hospital of People's Liberation Army, Guangzhou, Guangdong 510620; \\ Departments of ${ }^{3}$ Oncology and ${ }^{4}$ Clinical Medical Research, Shenzhen People's Hospital, \\ The Second Clinical Medical College of Jinan University, Shenzhen, Guangdong 518000, P.R. China
}

Received August 28, 2016; Accepted September 29, 2017

DOI: $10.3892 / 01.2018 .7803$

\begin{abstract}
Breast cancer is the most frequently diagnosed malignancy in women. However, the molecular mechanisms underlying breast cancer pathogenesis are not fully understood. The present study examined the role of miR-433 in breast cancer and investigated its underlying molecular mechanisms of action. Reverse transcription-quantitative polymerase chain reaction and western blot analysis were performed to analyze the level of microRNA (miRNA/miR)/mRNA and protein expression, respectively. Additionally, MTT assay was used to determined cell proliferation and viability. Cell apoptosis was measured by flow cytometry. A dual-luciferase reporter assay was used to confirm the identity of the downstream target of miR-433. The results revealed that miR-433 was downregulated in breast cancer tissues and cell lines. Overexpression of miR-433 inhibited cell proliferation and cell viability in BT-549 cells, whereas downregulation of miR-433 increased cell proliferation and cell viability in MDA-MB-231 cells. Further flow cytometry analysis revealed that miR-433 was able to induce apoptosis and also alter the levels of proteins expression of B-cell lymphoma-2 and Bcl-associated X. Bioinformatics analysis showed that RAC- $\gamma$ serine/threonine-protein kinase (AKT3) was one of the downstream targets of miR-433, and luciferase reporter assay further confirmed that AKT3 is a direct target of miR-433. The knockdown of AKT3 was able to inhibit proliferation and viability in BT-549 cells. Overexpression of AKT3 prevented
\end{abstract}

Correspondence to: Dr Xiaolei $\mathrm{Hu}$ or Dr Changsheng Ye, Department of Breast Surgery, Nanfang Hospital, Southern Medical University, 1838 Guangzhou Road, Guangzhou, Guangdong 510515, P.R. China

E-mail: frozenme@126.com

E-mail: chshye@126.com

*Contributed equally

Key words: breast cancer, microRNA-433, cell proliferation, cell viability, apoptosis, RAC- $\gamma$ serine/threonine-protein kinase the inhibitory effects of miR-433 on proliferation and viability in BT-549 cells. The level of AKT3 mRNA expression was upregulated in breast cancer tissues compared with normal tissues and was inversely correlated with miR-433 expression levels. In summary, the results of the present study results indicate that the tumor-suppressive role of miR-433 may be mediated by regulating AKT3. miR-433 may therefore serve as a potential therapeutic target for breast cancer.

\section{Introduction}

Breast cancer is the most frequently diagnosed malignancy in women worldwide and poses a serious threat to women's health (1). Breast cancer is the second-leading cause of cancer-associated mortality in 2015 , accounting for $\sim 11 \%$ of the total cancer mortalities in women $(2,3)$. Although substantial progress has been made in surgical management and chemotherapy treatment, the rate of relapse in patients with advanced breast cancer remains high (4-6). It is therefore essential to investigate further the molecular mechanisms underlying breast cancer development.

Previous findings concerning microRNAs (miRNAs/miRs) have substantially broadened knowledge concerning cancer pathogenesis $(7,8)$. MiRNAs are a class of short non-coding RNA of 20-22 nucleotides that can bind to complementary sequences in the $3^{\prime}$ untranslated region (3'UTR) of mRNA to regulate gene expression. Therefore, miRNAs are involved in a number of cellular processes, including proliferation, apoptosis, development and differentiation (9). Numerous studies have demonstrated that miRNAs can perform oncogenic or tumor suppressive roles in various types of cancers. Previous studies have demonstrated the functional roles of miR-433 in several types of cancer, including retinoblastoma (10), ovarian cancer (11), bladder cancer (11), oral squamous cell carcinoma (OSCC) (12), gastric cancer (13), hepatocellular carcinoma (HCC) (14) and lung cancer (15). The downstream targets of miR-433 have been revealed to be strongly associated with cancer development, including Notch1, cAMP responsive element binding protein 1 (CREB1), paired box protein Pax-6 (PAX6), histone deacetylase 6 (HDAC6), kirsten rat sarcoma viral oncogene homolog and hepatocyte growth factor receptor (c-Met) $(10,12,13,16)$. MiR-433 expression was found 
to be downregulated in these cancer types, and miR-433 was demonstrated to function as a tumor suppressor by inhibiting cell proliferation, migration and differentiation $(10,12-14,16)$. However, to the best of our knowledge, the role of miR-433 in breast cancer development is largely unknown.

In the present study, miR-433 was downregulated in breast cancer tissues and breast cancer cell lines. In vitro functional assay indicated that miR-433 was able to inhibit cell proliferation, reduce cell viability and also decrease apoptosis in breast cancer cell lines. It was revealed that $\mathrm{RAC}-\gamma$ serine/threonine-protein kinase (AKT3) is a direct target of miR-433 in breast cancer cell lines. More importantly, analysis of clinical specimens further indicated that the level of miR-433 expression was inversely correlated with AKT3 expression in breast cancer tissues.

\section{Materials and methods}

Human clinical samples. Paired breast cancer and adjacent normal breast cancer tissues $(\sim 5 \mathrm{~cm}$ away from cancerous tissues) were obtained from 42 patients (mean age, 53 years old, age range: 27-68 years old) undergoing surgery in Nanfang Hospital (Guangzhou, China). The clinical samples were collected between July 2012 and December 2015. Informed consent was obtained from all patients enrolled in the present study, and all clinical studies were approved by the Ethics Committee of Nanfang Hospital. All the collected samples were snap-frozen for further reverse transcription-quantitative polymerase chain reaction (RT-qPCR) analysis.

Cell lines and cell culture. The human breast epithelial MCF-10A cell line, and breast cancer cell lines BT-549, MCF-7, MDA-MB-453 and MDA-MB-231 were purchased from the Shanghai Institute of Cell Biology (Shanghai, China). All cells were cultured in Dulbecco's modified Eagle's medium (DMEM; Thermo Fisher Scientific, Inc., Waltham, MA, USA) supplemented with $10 \%$ fetal bovine serum (FBS; Thermo Fisher Scientific, Inc.) under a humidified atmosphere of 5\% $\mathrm{CO}_{2}$ at $37^{\circ} \mathrm{C}$.

RNA isolation and $R T-q P C R$. miRNA and mRNA were extracted from clinical samples and cell lines using TRIzol reagent (Takara Biotechnology Co., Ltd., Dalian, China), according to the manufacturer's protocol. MiRNAs were reverse transcribed into cDNA using the One Step PrimeScript miRNA cDNA Synthesis kit (Takara Biotechnology Co., Ltd., Dailan, China), and mRNA was reverse transcribed into cDNA using the PrimeScript RT Reagent kit (Takara Biotechnology Co., Ltd.). RT-qPCR was performed by using the SYBR Green PCR kit (Takara Biotechnology Co., Ltd.) and the ABI 7500 FAST Real-Time PCR system (Applied Biosystems, Thermo Fisher Scientific, Inc.). The sequences of the primers used are as follows: miR-433 forward, 5'-TGCGGTACGGTG AGCTGTC-3' and reverse, 5'-CCAGTGCAGGGTCCGAGG T-3'; AKT3 forward, 5'-ATGAGCGATGTTACCATTGT-3' and reverse, 5'-CAGTCTGTCTGCTACAGCCTGGATA-3'; U6 forward: 5'-CGCTTCGGCAGCACATATAC-3', reverse: 5'-TTCACGAATTTGCGTGTCAT-3'; GAPDH forward, 5'-GGTGAAGGTCGGAGTCAACG-3', and reverse: 5'-CAA AGTTGTCATGGATGHACC-3'. The qPCR thermocycling conditions used were $95^{\circ} \mathrm{C}$ for $30 \mathrm{sec} ; 40$ cycles of $95^{\circ} \mathrm{C}$ for $5 \mathrm{sec}$ and $60^{\circ} \mathrm{C}$ for $30 \mathrm{sec} ; 95^{\circ} \mathrm{C}$ for $15 \mathrm{sec}, 60^{\circ} \mathrm{C}$ for $60 \mathrm{sec}$ and $95^{\circ} \mathrm{C}$ for $15 \mathrm{sec}$. The relative expression level of miR-433 and AKT3 mRNA were calculated using $2^{-\Delta \Delta \mathrm{Cq}}$ method (17) following normalization to U6 and GAPDH, respectively. All the experiments were performed in triplicates.

Reagents and transfection. The miR-433 mimics, and miR-433 inhibitors (anti-miR-433), as well as respective controls (miR-Ctrl and anti-miR-Ctrl), and the small interfering RNA (siRNA) targeting AKT3 (siAKT3), as well as its negative control (siNC), were obtained from Shanghai GeneChem, Inc. (Shanghai, China). The pGCL and pGCL-AKT3 plasmids were purchased from Shanghai GeneChem Co., Ltd. (Shanghai, China). In vitro transfection of these oligonucleotides $(50 \mathrm{nM})$ was performed using Lipofectamine 2000 (Invitrogen; Thermo Fisher Scientific, Inc., Waltham, MA, USA) in accordance with the manufacturer's instructions. At $48 \mathrm{~h}$ post-transfection, cells were processed for further experimentation.

Cell proliferation assay. Breast cancer cells (BT-549 and MDA-MB-231) were plated in 96-well plates overnight, and the cells were transfected with $50 \mathrm{nM}$ miR-433 mimics, or anti-miR-433, or their respective controls (miR-Ctrl, anti-miR-Ctrl) using Lipofectamine 2000 reagent. At $48 \mathrm{~h}$ post-transfection, cells were further cultured in DMEM supplemented with $10 \%$ FBS for $0,24,48$ or $72 \mathrm{~h}$ prior to the addition of MTT to each well. Following incubation with MTT for $4 \mathrm{~h}$ at $37^{\circ} \mathrm{C}$, MTT solution was removed, and dimethyl sulfoxide (DMSO) was added to each well. Absorbance was then measured at $490 \mathrm{~nm}$ using a spectrophotometer. For the AKT3 siRNA study and rescue experiments, the cells were transfected with siAKT3 or the negative control (siNC), miR-Ctrl with pGCL, miR-433 mimics + pGCL or miR-433 mimcis + pGCL-AKT3. A total of $48 \mathrm{~h}$ after transfection, the cells were further cultured for $48 \mathrm{~h}$ at $37^{\circ} \mathrm{C}$ and the MTT assay was performed as aforementioned.

Cell viability assay. Breast cancer cells (BT-549 and MDA-MB-231) were plated in 96-well plates overnight, and the cells were transfected with miR-433 mimics, anti-miR-433, miR-Ctrl or anti-miR-Ctrl. A total of $48 \mathrm{~h}$ after transfection, the cells were further cultured in serum-free DMEM for 0, 24, 48 or $72 \mathrm{~h}$ prior to the addition of MTT to each well. Following incubation with MTT for $4 \mathrm{~h}$ at $37^{\circ} \mathrm{C}$, the MTT solution was removed and DMSO was added to each well followed by measuring the absorbance at $490 \mathrm{~nm}$ using a spectrophotometer. For the AKT3 siRNA study and rescue experiment, the cells were transfected with siAKT3 or the negative control (siNC), miR-Ctrl + pGCL, miR-433 mimics + pGCL, or miR-433 mimcis + pGCL-AKT3, and $48 \mathrm{~h}$ after transfection, the cells were further cultured for $48 \mathrm{~h}$ at $37^{\circ} \mathrm{C}$ before MTT assay was conducted as aforementioned.

Luciferase reporter assay. The predicted targets of miR-433 were analyzed by using TargetScan (version 7.1), and AKT3 was found to one of the targets of miR-433. The entire human AKT3 3'UTR, harboring the miR-433 target sequence as well as the mutated (MUT) seed sequence, were purchased from Guangzhou RiboBio Co., Ltd. (Guangzhou, China). The 

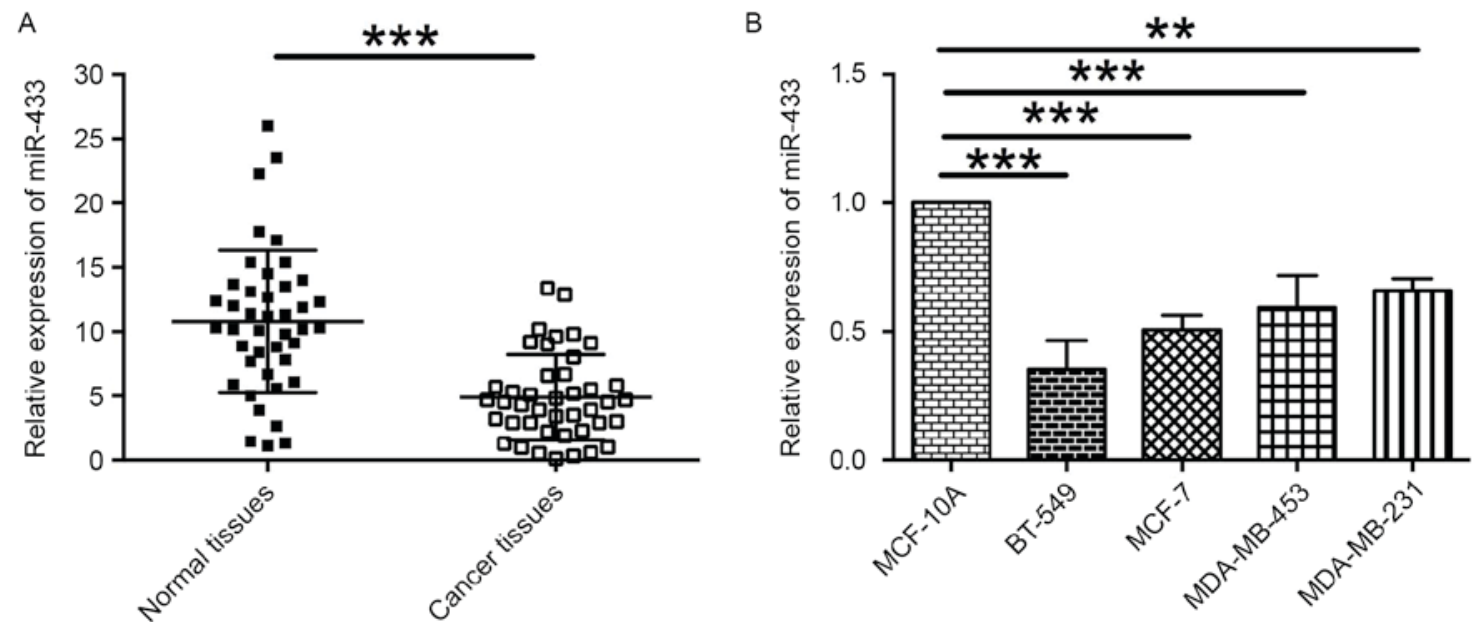

Figure 1. Downregulation of miR-433 in breast cancer tissues and cell lines. (A) The miR-433 expression levels in normal breast cancer tissues (n=42) and breast cancer tissues $(\mathrm{n}=42$ ) were examined by RT-qPCR (paired t-test). (B) The miR-433 expression levels in the normal breast MCF-10A cell line and breast cancer BT-549, MCF-7, MDA-MB-453, MDA-MB-231 cell lines were determined by RT-qPCR; $n=3$ (one-way analysis of variance followed by Dunnett's multiple comparison test). ${ }^{* *} \mathrm{P}<0.01,{ }^{* * *} \mathrm{P}<0.001$. miR, microRNA; RT-qPCR, reverse transcription-quantitative polymerase chain reaction

AKT3 3'UTR reporter was generated by inserting the entire wild-type (WT) 3'UTR or MUT 3'UTR of AKT3 mRNA into psiCHECK-2 vector (Promega Corporation, Madison, WI, USA). For the luciferase reporter assay, the cells (BT-549 and MDA-MB-231) were co-transfected with the AKT3 3'UTR reporter and the miR-433 mimics, anti-miR-433 or their respective controls (miR-Ctrl, anti-miR-Ctrl) using the Lipofectamine 2000 reagent, and $48 \mathrm{~h}$ following transfection, firefly and Renilla luciferase activities were measured by using the Dual Luciferase Assay kit (Promega Corporation, Madison, WI, USA).

Cell apoptosis analysis. Breast cancer cells (BT-549 and MDA-MB-231) were plated in 6-well plates overnight, and the cells were then transfected with miR-433 mimics, anti-miR-433 or respective controls (miR-Ctrl and anti-miR-Ctrl). At $48 \mathrm{~h}$ after transfection, the cells were harvested and processed, and double staining with annexin V-fluorescein isothiocyanate (FITC) and propidium iodide was performed using the Annexin V-FITC Apoptosis Detection kit (BD Biosciences, Franklin Lakes, NJ, USA) according to the manufacturer's protocol. Cell apoptosis was analyzed with a FACScan flow cytometer (BD Biosciences), and the cell apoptotic rates were analyzed by the BD CellQuest Pro $^{\mathrm{TM}}$ Software (Version 5.1; BD Biosciences).

Western blot analysis. Proteins from breast cancer cells were extracted using a modified Radioimmunoprecipitation Assay buffer (Sigma-Aldrich; Merck KGaA, Darmstadt, Germany) with proteinase inhibitor cocktail (Complete mini; Roche Applied Science, Penzberg, Germany). The concentration of protein was measured using the BCA kit (Bio-Rad Laboratories, Inc., Hercules, CA, USA) Protein lysates (30 $\mu \mathrm{g})$ were separated on $10 \%$ SDS-PAGE and transferred to polyvinylidene difluoride membranes. The membranes were blocked with $5 \%$ skimmed milk for $1 \mathrm{~h}$ at room temperature, followed by incubating with the following primary antibodies: Rabbit polyclonal anti-B-cell lymphoma 2 (Bcl-2) antibody (1:1,500; cat. no. ab59348; Abcam, Cambridge, USA), rabbit polyclonal
anti-Bcl-associated X (Bax) antibody (1:1,000; cat. no. ab53154; Abcam), rabbit polyclonal anti-AKT3 antibody $(1: 1,000$, cat. no. ab189643; Abcam) and rabbit polyclonal anti-GAPDH antibody (1:2,000, cat. no. ab9485; Abcam) overnight at $4^{\circ} \mathrm{C}$. The membranes were subsequently incubated with horseradish peroxidase conjugated secondary antibody $(1: 2,000$, cat. no. ab205718; Abcam) and visualized with enhanced chemiluminescence reagent (GE Healthcare Life Sciences, Little Chalfont, UK) according to the manufacturer's protocol.

Statistical analysis. All data are presented as the mean \pm standard deviation, and data analysis was performed using GraphPad Prism (version 6.0; GraphPad Software, Inc., La Jolla, CA, USA). Significant differences between groups for clinical samples were analyzed by paired t-test. For in vitro functional assay, significant differences were analyzed by one-way analysis of variance followed by Dunnett's multiple comparison test or unpaired t-test. The correlation between miR-433 levels and AKT3 mRNA levels in breast cancer tissues was analyzed by Spearman's correlation test. $\mathrm{P}<0.05$ was considered to indicate a statistically significant difference.

\section{Results}

miR-433 expression is downregulated in breast cancer tissues and breast cancer cell lines. To assess the role of miR-433 in breast cancer, the levels of miR-433 expression in 42 paired breast cancer and normal adjacent breast tissues were examined by RT-qPCR. The results revealed that the level of miR-433 expression was significantly lower in cancer tissues compared with normal adjacent breast tissues (Fig. 1A). In addition, the relative expression levels of miR-433 in different breast cancer cells lines were also determined. It was indicated that the levels of miR-433 expression in the breast cancer cell lines BT-549, MCF-7, MDA-MB-453 and MDA-MB-231 was downregulated when compared with the expression level of miR-433 in normal breast cell line, MCF-10A (Fig. 1B). These data indicate that miR-433 may be a tumor suppressor in breast cancer. 

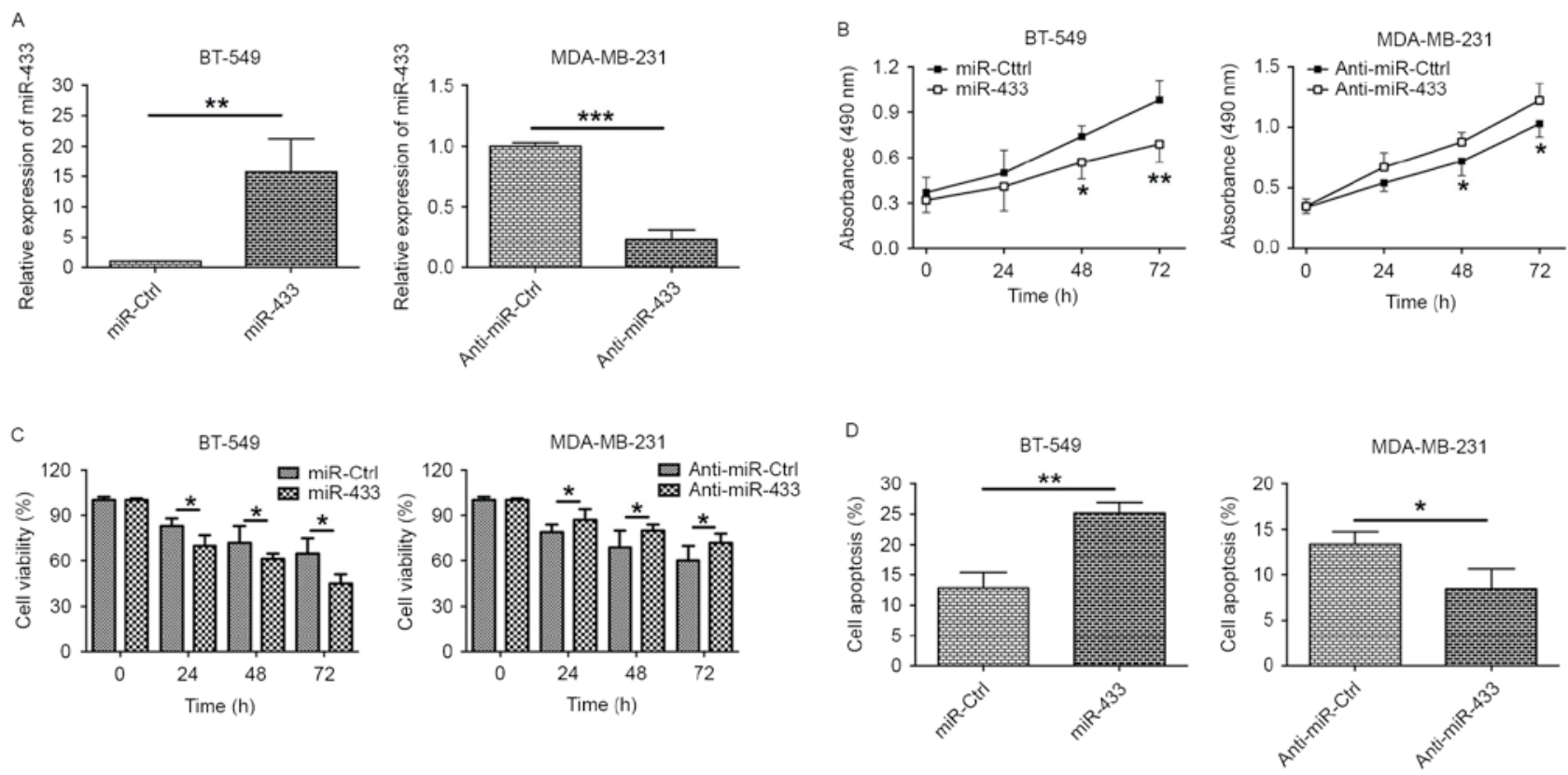

$\mathrm{E}$

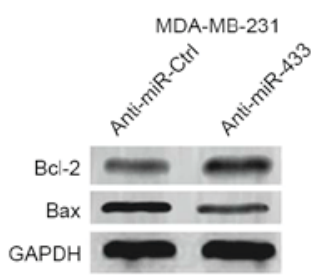

Figure 2. miR-433 inhibits proliferation and induces apoptosis in breast cancer cell lines. BT-549 cells were transfected with miR-Ctrl or miR-433 mimics. MDA-MB-231 cells were transfected with anti-miR-Ctrl or miR-433 inhibitors (anti-miR-433). A total of $24 \mathrm{~h}$ post-transfection, (A) the expression levels of miR-433 were determined by reverse transcription-quantitative polymerase chain reaction; (B) Cell proliferation was determined by MTT assay. (C) Cell viability was examined by cell viability assay. (D) Cell apoptosis was determined by flow cytometry. (E) Protein expression levels of Bcl-2 and Bax were examined by western blotting assay. ${ }^{*} \mathrm{P}<0.05 ;{ }^{* *} \mathrm{P}<0.01 ;{ }^{* * *} \mathrm{P}<0.001$ (unpaired t-test; $\mathrm{n}=3$ ). miR, microRNA; miR-Ctrl, control scrambled miRs; anti-miR-433, miR-433 inhibitor; Bcl-2, B-cell lymphoma 2; Bax, Bcl-associated X.

miR-433 inhibits proliferation in breast cancer cell lines. To gain insights into the underlying mechanisms of action of miR-433 in breast cancer, in vitro functional assays including MTT and cell viability assays were performed. RT-qPCR results revealed that the expression level of miR-433 was increased $>10$ fold in BT-549 cells transfected with miR-433 mimics, while the expression level of miR-433 was significantly decreased in MDA-MB-231 cells transfected with anti-miR-433 (Fig. 2A). The MTT assay revealed that BT-549 cells transfected with miR-433 mimics exhibited lower proliferative activity when compared with cells transfected with miR-Ctrl. Additionally, the downregulation of miR-433 in MDA-MB-231 cells by transfection with anti-miR-433 was able to increase cell proliferation (Fig. 2B). The viability of BT-549 cells transfected with miR-Ctrl or miR-433 mimics and MDA-MB-231 cells transfected with anti-miR-Ctrl or anti-miR-433 was also examined. As shown in Fig. 2C, transfection with miR-433 mimic in BT-549 cells reduced cell viability when compared with miR-Ctrl transfection, whereas MDA-MB-231 cells transfected with anti-miR-433 exhibited a higher cell viability when compared with cells transfected with anti-miR-Ctrl (Fig. 2C). These results indicate that miR-433 exerts a tumor suppressive function in breast cancer cells.

miR-433 induces apoptosis in breast cancer cell lines. To examine the role of miR-433 on cell apoptosis, flow cytometry and western blotting experiments were performed. Flow cytometry results demonstrated that transfection of BT-549 cells with the miR-433 mimic significantly increased the proportion of apoptotic cells when compared with cells transfected with miR-Ctrl, whereas the proportion of apoptotic MDA-MB-231 cells transfected with anti-miR-433 was significantly lower compared with cells transfected with anti-miR-Ctrl (Fig. 2D). Expression levels of the apoptosis-associated proteins, including Bcl-2 and Bax, were examined. Overexpression of miR-433 in BT-549 cells decreased the level of Bcl-2 protein expression and increased the level of Bax protein expression, whereas downregulation of miR-433 in MDA-MB-231 cells upregulated Bcl-2 expression and downregulated Bax expression (Fig. 2E).

AKT3 is a direct target of miR-433. To predict the downstream targets of miR-433, the predicted targets were analyzed by using TargetScan (version 7.1). Of the predicted targets, AKT3 was selected for further experiments owing to its known role in cancer development (18). To confirm that AKT3 was a target of miR-433, AKT3 3'UTR luciferase reporter plasmids containing WT or MUT potential binding sites for miR-433 were constructed and a dual-luciferase reporter assay was conducted (Fig. 3A). The results of this assay revealed that overexpression of miR-433 significantly decreased luciferase activity in BT-549 cells transfected with plasmids containing 

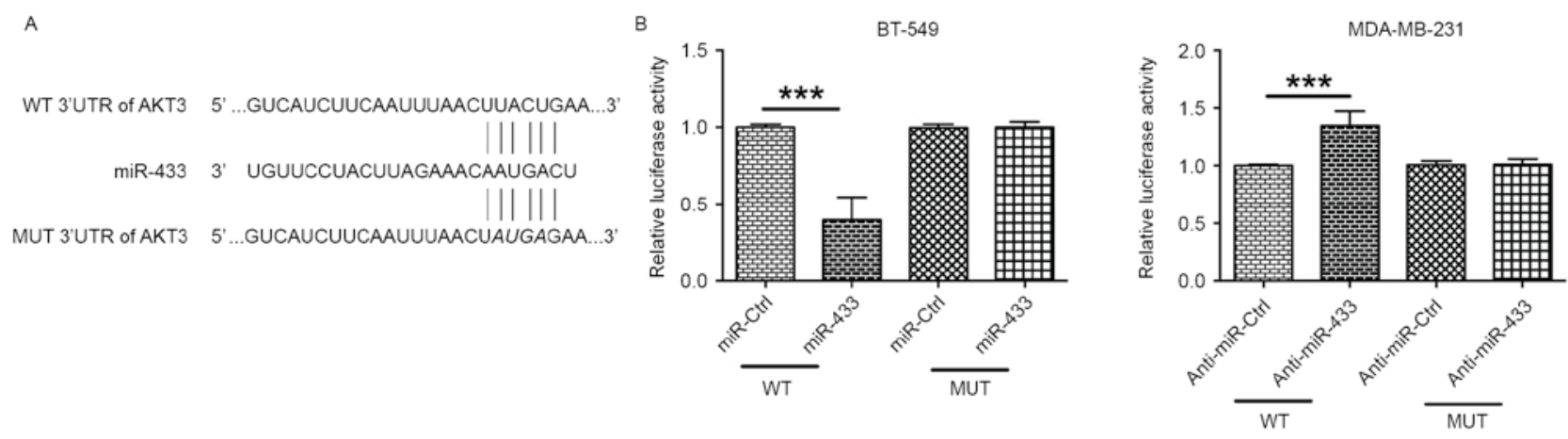

c
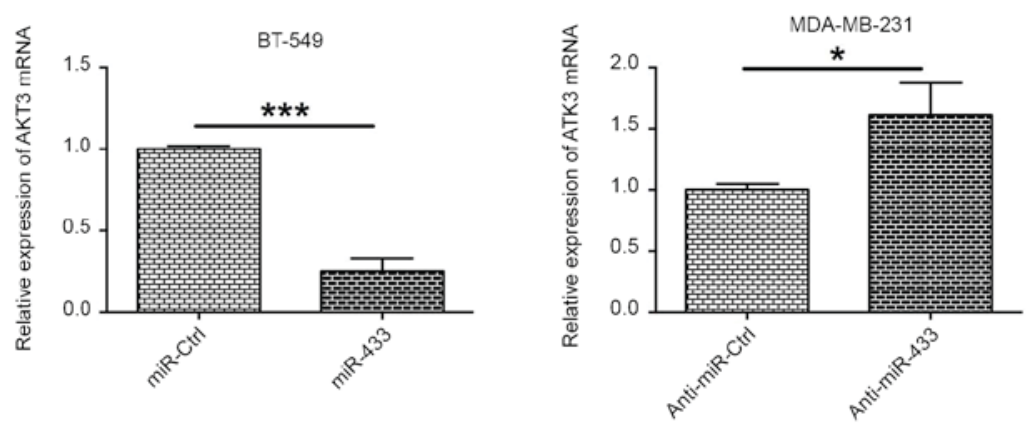

Figure 3. AKT3 is a direct target of miR-433. (A) Schematic representation of miR-433 putative binding sites in the 3'UTR of AKT3 mRNA, and the mutations in the 3'UTR region of AKT3. (B) In BT-549 cells, the relative luciferase activities were measured following the co-transfection of the cells with miR-Ctrl (or miR-433 mimics) and WT 3'UTR (or MUT 3'UTR). In MDA-MB-231, the relative luciferase activities were measured following the co-transfection of the cells with anti-miR-Ctrl (or anti-miR-433) and WT 3'UTR (or MUT 3'UTR). (C) The relative expression of AKT3 mRNA in BT-549 cells transfected with miR-Ctrl or miR-433, and in MDA-MB-231 cells transfected with anti-miR-Ctrl or anti-miR-433 was examined by reverse transcription-quantitative polymerase chain reaction. ${ }^{*} \mathrm{P}<0.05,{ }^{* * *} \mathrm{P}<0.001$ (unpaired $\mathrm{t}$-test; $\mathrm{n}=3$ ). AKT3, RAC- $\gamma$ serine/threonine-protein kinase; miR, microRNA; $3^{\prime} \mathrm{UTR}, 3^{\prime}$ untranslated region; WT, wild-type; MUT, mutated; miR-Ctrl, control scrambled microRNA.

the WT 3'UTR of AKT3, but not in cells transfected with plasmids containing the MUT 3'UTR of AKT3 (Fig. 3B). Downregulation of miR-433 by anti-miR-433 transfection significantly increased luciferase activity in MDA-MB-231 cells transfected with plasmids containing the WT 3'UTR of AKT3, but not in cells transfected with plasmids containing the MUT 3'UTR of AKT3 (Fig. 3B). In addition, BT-549 cells transfected with miR-433 mimics expressed lower levels of ATK3 mRNA compared with cells transfected with miR-Ctrl (Fig. 3C), and MDA-MB-231 cells transfected with anti-miR-433 exhibited increased AKT3 mRNA the expression levels of AKT3 mRNA when compared to with cells transfected with anti-miR-Ctrl (Fig. 3C). These results suggest that AKT3 may be a direct target of miR-433.

Inverse correlation between the expression levels of miR-433 and AKT3. To further examine the role of AKT3 in breast cancer cells, a siRNA experiment to determine the effect of AKT3-knockdown on proliferation and cell viability of breast cancer cells was performed. As shown in Fig. 4A and B, AKT3-knockdown in BT-549 cells by transfection with siAKT3 significantly decreased the mRNA and protein expression levels (Fig. 4A and B). Knockdown of AKT3 in BT-549 cells was able to decrease cell proliferation and viability (Fig. 4C and D). To further confirm that ATK3 is a downstream target of miR-433, a rescue experiment was also performed by transfecting BT-549 cells with miR-433 mimics and AKT3-expressing vectors. The results revealed that overexpression of AKT3 in miR-433-transfected BT-549 cells significantly prevented the inhibitory effect of miR-433 on cell proliferation and cell viability (Fig. 4E and F). Analysis of clinical samples revealed that the level of AKT3 mRNA expression in breast cancer tissues was significantly higher compared with adjacent normal breast cancer tissues (Fig. 4G), and Spearman's correlation analysis demonstrated that miR-433 expression level was inversely correlated with the level of AKT3 mRNA expression (Fig. 4H).

\section{Discussion}

Recently, substantial effort has been made to improve diagnostic techniques and therapeutic approach for breast cancer. However, the limited efficacy of novel therapeutics has become a major obstacle owing to the inadequate understanding of molecular mechanisms underlying breast cancer pathogenesis (4-6). The present study demonstrated the tumor suppressive role of miR-433 in breast cancer cells. miR-433 expression was found to be downregulated in breast cancer tissues and breast cancer cell lines, and miR-433 overexpression was revealed to inhibit cell proliferation and viability. Additionally, overexpression of miR-433 was able to induce apoptosis possibly via targeting of AKT3 in breast cancer cells. Clinical sample analysis further confirmed that miR-433 and AKT3 expression levels were inversely correlated in breast cancer tissues.

The functional role of miR-433 has been determined in various types of cancer. miR- 433 has been demonstrated to be downregulated in several types of cancer tissue, including 
A

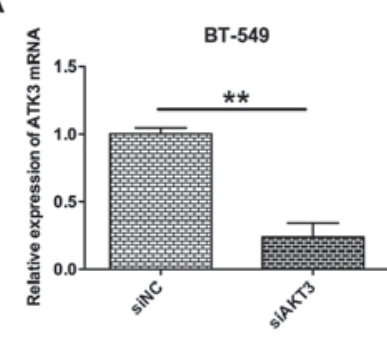

E

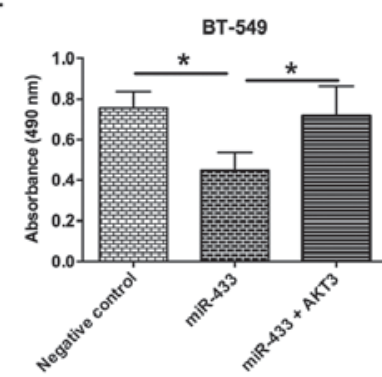

B
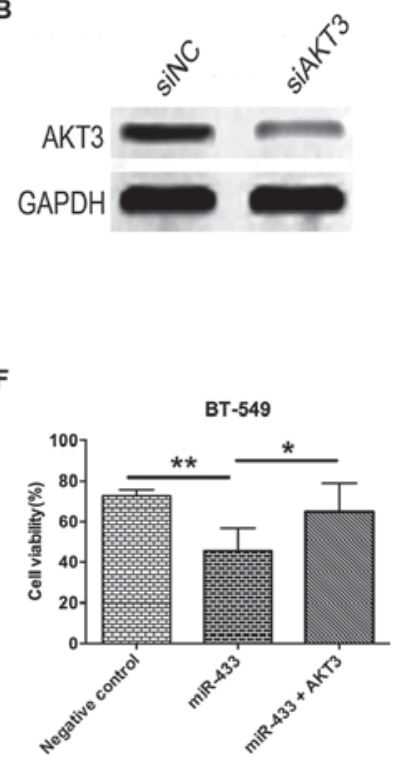

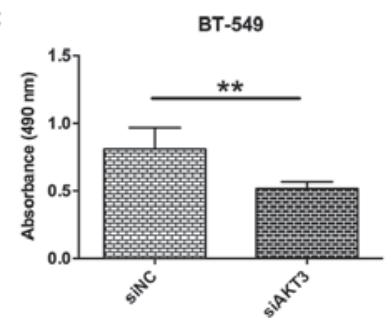

G

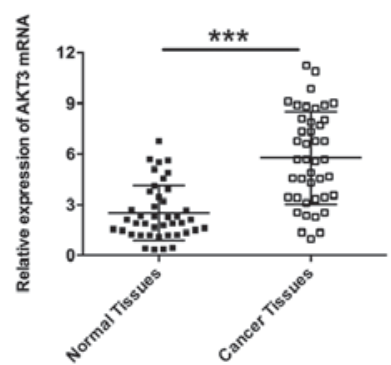

D

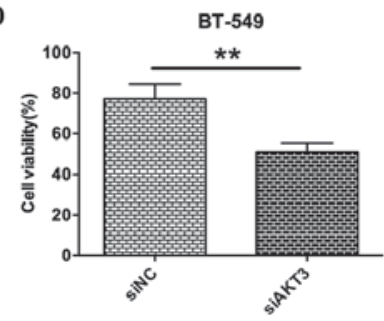

H

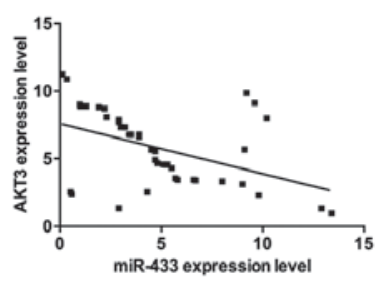

Figure 4. Inverse correlation between the expression levels of miR-433 and AKT3. The levels of AKT3 mRNA and protein expression in BT-549 cells transfected with siNC or siAKT3 were detected by (A) RT-qPCR and (B) western blotting, respectively (unpaired t-test; $\mathrm{n}=3$ ). (C) Cell proliferation and (D) viability was detected in BT-549 cells transfected with siNC or siAKT3 (unpaired t-test; $\mathrm{n}=3$ ). (E) Cell proliferation and (F) viability was detected in BT-549 cells transfected with miR-Ctrl + pGCL, miR-433 mimics + pGCL or miR-433 mimics + pGCL-AKT3 (one-way analysis of variance followed by Dunnett's multiple comparison test). (G) The expression levels of AKT3 mRNA in normal breast cancer tissues $(n=42)$ and breast cancer tissues $(n=42)$ were examined by RT-qPCR (paired t-test). (H) The correlation between miR-433 levels and AKT3 mRNA levels in breast cancer tissues was analyzed by Spearman's correlation test $(\mathrm{P}<0.01 ; \mathrm{r}=-0.4428){ }^{* *} \mathrm{P}<0.01,{ }^{* * * *} \mathrm{P}<0.001$. miR, microRNA; AKT3, RAC- $\gamma$ serine/threonine-protein kinase; siNC, negative control small interfering RNA; RT-qPCR, reverse transcription-quantitative polymerase chain reaction; miR-Ctrl, control scrambled microRNA.

retinoblastoma, bladder cancer, OSCC, gastric cancer and HCC $(10,12-14,16)$. Consistent with this, the results of the present study also demonstrated the downregulation of miR-433 in breast cancer tissues, which suggests that miR-433 may be tumor-suppressive in breast cancer tissues. To gain further understanding of miR-433 in breast cancer development, MTT assay was performed in the present study to assess cell viability and proliferation, and it was indicated that transfection with miR-433 was bale to inhibit cell proliferation and reduce the viability of breast cancer cells. These findings were in agreement with those of previous studies, as miR-433 was shown to negatively regulate cell proliferation, migration and invasion in retinoblastoma cells (10). Exogenous expression of miR-433 was able to significantly inhibit cell proliferation, colony formation, migration and invasion in bladder cancer cells (16). Restoring miR-433 expression in OSCC cells dramatically suppressed cell growth, invasion and migration, (12) and ectopic expression of miR-433 inhibited cell proliferation, cell cycle progression, cell migration and invasion in gastric cancer cells (19). Collectively, these results indicate that $\mathrm{miR}-433$ has a tumor-suppressive role in cancer cells in vitro. In addition, overexpression of miR-433 induced cell apoptosis and altered the expression levels of apoptosis mediators (Bcl-2 and Bax). miR-433 induced cell cycle arrest and cell apoptosis in retinoblastoma cells (10), and miR-433 accelerated apoptosis in human dental pulp cells (20). Therefore, the results of these previous studies indicate that the inhibitory effect of miR-433 on breast cancer cell proliferation and cell viability may involve the acceleration of cell apoptosis.

AKT is a key mediator of the phosphoinositide 3-kinase (PI3K) pathway, and was found to regulate breast cancer progression and metastasis by promoting migration and invasion $(21,22)$. Activation of the PI3K/AKT signaling pathway is observed in $>80 \%$ of breast cancer patients (23). AKT3 is one of the three isoforms of AKT (AKT1, AKT2, and AKT3), and has been demonstrated to serve notable roles in breast cancer development (24-29). AKT3 amplification has been suggested to represent a potentially relevant oncogenic event in the subset of triple-negative breast cancer (TNBC) (29). Downregulation of AKT3 inhibited the growth of TNBC cell lines in 3D spheroid cultures and in mouse xenograft models (24), whereas upregulation of AKT3 conferred resistance to the AKT inhibitor MK2206 in breast cancer, and suppression of AKT3 by miR-489 increases chemosensitivity in breast cancer (28).

In the present study, it was found that AKT3 is a direct target of miR-433, which was confirmed by luciferase reporter assay. The knockdown of AKT3 was also able to significantly inhibit proliferation and viability of breast cancer cells. The level of AKT3 expression was inversely correlated with miR-433 expression in breast cancer tissues. Collectively, these data indicate that miR-433 was able to inhibit cell proliferation and cell viability by modulating AKT3. However, caution must be exercised as miR-433 may have downstream targets other than AKT3. Previous studies have found that miR-433 targets Notch1, CREB1, PAX6, HDAC6, KRAS and c-Met in other types of cancer $(10,12,13,16)$. Therefore, future studies must assess other targets of miR-433 in breast cancer.

In summary, in the present study, it was revealed that miR-433 was downregulated in breast cancer tissues and cell lines. To the best of our knowledge, the present study is the first to reveal that miR-433 may function as a tumor suppressor by targeting AKT3 in breast cancer cells. Therefore miR-433 may be a potential biomarker for breast cancer diagnosis and may serve as a novel target for breast cancer therapy. 


\section{Acknowledgements}

The present study was supported by a grant from the Science and Technology Planning Project of Guangdong Province (grant no. 2015B090904007).

\section{References}

1. Rudolph A, Chang-Claude J and Schmidt MK: Gene-environment interaction and risk of breast cancer. Br J Cancer 114: 125-133, 2016

2. Li C, Yang L, Zhang D and Jiang W: Systematic review and meta-analysis suggest that dietary cholesterol intake increases risk of breast cancer. Nutr Res 36: 627-635, 2016.

3. Srivastava S, Reid BJ, Ghosh S and Kramer BS: Research needs for understanding the biology of overdiagnosis in cancer screening. J Cell Physiol 231: 1870-1875, 2016.

4. Golubnitschaja O, Debald M, Yeghiazaryan K, Kuhn W, Pešta M, Costigliola V and Grech G: Breast cancer epidemic in the early twenty-first century: Evaluation of risk factors, cumulative questionnaires and recommendations for preventive measures Tumour Biol 37: 12941-12957, 2016.

5. Sonnenblick A, Pondé N and Piccart M: Metastatic breast cancer: The Odyssey of personalization. Mol Oncol 10: 1147-1159, 2016.

6. Tzanninis IG, Kotteas EA, Ntanasis-Stathopoulos I, Kontogianni P and Fotopoulos G: Management and outcomes in metaplastic breast cancer. Clin Breast Cancer 16: 437-443, 2016.

7. Hemmatzadeh M, Mohammadi H, Jadidi-Niaragh F, Asghari $\mathrm{F}$ and Yousefi M: The role of oncomirs in the pathogenesis and treatment of breast cancer. Biomed Pharmacother 78: 129-139, 2016.

8. Pinweha P, Rattanapornsompong K, Charoensawan V and Jitrapakdee S: MicroRNAs and oncogenic transcriptional regulatory networks controlling metabolic reprogramming in cancers Comput Struct Biotechnol J 14: 223-233, 2016.

9. Leung AK: The Whereabouts of microRNA Actions: Cytoplasm and beyond. Trends Cell Biol 25: 601-610, 2015.

10. Li X, Yang L, Shuai T, Piao T and Wang R: MiR-433 inhibits retinoblastoma malignancy by suppressing Notch1 and PAX6 expression. Biomed Pharmacother 82: 247-255, 2016.

11. Weiner-Gorzel K, Dempsey E, Milewska M, McGoldrick A, Toh V, Walsh A, Lindsay S, Gubbins L, Cannon A, Sharpe D, et al: Overexpression of the microRNA miR-433 promotes resistance to paclitaxel through the induction of cellular senescence in ovarian cancer cells. Cancer Med 4: 745-758, 2015.

12. Wang XC, Ma Y, Meng PS, Han JL, Yu HY and Bi LJ: miR-433 inhibits oral squamous cell carcinoma (OSCC) cell growth and metastasis by targeting HDAC6. Oral Oncol 51: 674-682, 2015.

13. Guo LH, Li H, Wang F, Yu J and He JS: The tumor suppressor roles of miR-433 and miR-127 in gastric cancer. Int J Mol Sci 14: 14171-14184, 2013.

14. Tan Y, Ge G, Pan T, Wen D, Chen L, Yu X, Zhou X and Gan J: A serum microRNA panel as potential biomarkers for hepatocellular carcinoma related with hepatitis B virus. PLoS One 9: e107986, 2014.
15. Del Vescovo V, Meier T, Inga A, Denti MA and Borlak J: A cross-platform comparison of affymetrix and Agilent microarrays reveals discordant miRNA expression in lung tumors of c-Raf transgenic mice. PLoS One 8: e78870, 2013

16. Xu X, Zhu Y, Liang Z, Li S, Xu X, Wang X, Wu J, Hu Z, Meng S, Liu B, et al: c-Met and CREB1 are involved in miR-433-mediated inhibition of the epithelial-mesenchymal transition in bladder cancer by regulating Akt/GSK-3//Snail signaling. Cell Death Dis 7: e2088, 2016.

17. Livak KJ and Schmittgen TD: Analysis of relative gene expression data using real-time quantitative PCR and the 2(-Delta Delta C(T)) method. Methods 25: 402-408, 2001

18. Bellacosa A, Kumar CC, Di Cristofano A and Testa JR: Activation of AKT kinases in cancer: Implications for therapeutic targeting. Adv Cancer Res 94: 29-86, 2005.

19. Luo H, Zhang H, Zhang Z, Zhang X, Ning B, Guo J, Nie N, Liu B and Wu X: Down-regulated miR-9 and miR-433 in human gastric carcinoma. J Exp Clin Cancer Res 28: 82, 2009.

20. Wang K, Li L, Wu J, Qiu Q, Zhou F and Wu H: The different expression profiles of microRNAs in elderly and young human dental pulp and the role of miR-433 in human dental pulp cells. Mech Ageing Dev 146-148: 1-11, 2015.

21. Dillon RL and Muller WJ: Distinct biological roles for the akt family in mammary tumor progression. Cancer Res 70 : 4260-4264, 2010.

22. Chin YR and Toker A: Function of Akt/PKB signaling to cell motility, invasion and the tumor stroma in cancer. Cell Signal 21: 470-476, 2009.

23. Sultana A, Idress R, Naqvi ZA, Azam I, Khan S, Siddiqui AA and Lalani EN: Expression of the androgen receptor, pAkt, and pPTEN in breast cancer and their potential in prognostication. Transl Oncol: May 12, 2014 (Epub ahead of print).

24. Chin YR, Yoshida T, Marusyk A, Beck AH, Polyak K and Toker A: Targeting Akt3 signaling in triple-negative breast cancer. Cancer Res 74: 964-973, 2014.

25. Grottke A, Ewald F, Lange T, Nörz D, Herzberger C, Bach J, Grabinski N, Gräser L, Höppner F, Nashan B, et al: Downregulation of AKT3 increases migration and metastasis in triple negative breast cancer cells by upregulating S100A4. PLoS One 11: e0146370, 2016

26. Mosquera JM, Varma S, Pauli C, MacDonald TY, Yashinskie JJ, Varga Z, Sboner A, Moch H, Rubin MA and Shin SJ: MAGI3-AKT3 fusion in breast cancer amended. Nature 520: E11-E12, 2015

27. O'Hurley G, Daly E, O'Grady A, Cummins R, Quinn C, Flanagan L, Pierce A, Fan Y, Lynn MA, Rafferty M, et al: Investigation of molecular alterations of AKT-3 in triple-negative breast cancer. Histopathology 64: 660-670, 2014.

28. Stottrup C, Tsang T and Chin YR: Upregulation of AKT3 confers resistance to the AKT inhibitor MK2206 in breast cancer. Mol Cancer Ther 15: 1964-1974, 2016.

29. Waugh MG: Amplification of chromosome 1q genes encoding the phosphoinositide signalling enzymes PI4KB, AKT3, PIP5K1A and PI3KC2B in breast cancer. J Cancer 5: 790-796, 2014. 